Check for updates

Cite this: RSC Adv., 2017, 7, 20266

\title{
Studies of granular bed filter for dust removal in the process of coal pyrolysis by solid heat carrier
}

\author{
Ya-Qing Zhang, Peng Liang, (D) Juan Yu, Jia-Long Zhu and Xi-Zhuang Qin
}

Aiming at the optimization of the dust removal process in the poly-generation system of circulating fluidized bed (CFB) combustion/coal pyrolysis, a granular bed filter (GBF) is applied to the coal pyrolysis by solid heat carrier. By carrying out lab-scale experiments with a moving-bed pyrolyzer, the GBF model proposed by $\mathrm{C}$. Tien et al. was used to describe the dust removal process of the dust-contained volatiles which has high temperature and high viscosity. The model applicability for real pyrolysis volatiles was verified. The results show that, the particle size distribution of the dust uncollected by the GBF is relatively concentrated. The majority particle size of uncollected dust is less than $20 \mu \mathrm{m}$, the mass fraction is $\sim 80 \%$. The GBF is in a state of unsteady operation in the process of dust filtration. With the increase of operating time, the dust is gradually deposited in the GBF, then resulting in an increase of bed pressure drop. In the examined range, the calculational value of GBF efficiency is slightly lower than the experimental value. On the one hand, the filter media particles have a certain specific surface area, so that part of the dust-contained tar can be adsorbed on the filter media surface to achieve a better interception effect. On the other hand, the dust particle deposited in the GBF also plays an important role in dust capture. The $C$. Tien model results of the initial filtration efficiency can be used as the selection reference of the medium species and the physical properties of the GBF. The higher filter height, slower superficial gas velocity, and smaller size of medium are conducive to decrease the dust content in the pyrolysis products. This study can provide basic data for the dust removal process in the poly-generation system of CFB combustion/coal pyrolysis.

rsc.li/rsc-advances

\section{Introduction}

In recent years, the staged conversion of coal quality has become an important development direction for the clean and efficient utilization of coal resources. ${ }^{1}$ The moving-bed coal pyrolysis technology has been paid much attention by researchers because of its stable operation, uniform heating and easier reactor amplification..$^{2-4}$ Moreover, the circulating fluidized bed (CFB) combustion technology which is characterized by wide fuel flexibility, high processing load and low pollution has been developed greatly. ${ }^{5}$ This lays a foundation for the industrialization process of the poly-generation technology which combines CFB combustion with coal pyrolysis. ${ }^{6,7}$ In the poly-generation process, the hydrogen-rich component of coal is converted to gas and tar in the pyrolysis reactor, and the remaining carbon-rich component is fed into the CFB boiler to generate electricity and supply heat. Therefore the staged conversion of coal quality is realized. ${ }^{8-10}$

The poly-generation technology of CFB combustion/coal pyrolysis has broad application prospect. However, due to the

College of Chemical and Environmental Engineering, Shandong University of Science and Technology, Qingdao, Shandong 266590, P. R. China. E-mail: liangpeng202@ hotmail.com; Fax: +86 53286057718; Tel: +86 13678890728 circulating ash from CFB boiler is used as heat carrier, the dust content in pyrolysis volatiles is very high. The dust deposits in the tar will form intractable tar residues and then brings operating problems such as pipeline blockage and corrosion. ${ }^{11}$ Therefore, the dust removal performance of high-temperature pyrolysis volatiles affects directly on the tar quality and the stable operation of the poly-generation system. ${ }^{12,13}$ Moreover, in the currently developed technologies of other rapid coal pyrolysis, ${ }^{14}$ same problems are also common: dust is difficult to separate from the gas/tar, high dust content in tar, etc. Dust removal of coal pyrolysis volatiles has become one of the bottlenecks in the development of the fast pyrolysis process. For the coal pyrolysis volatiles, due to its characteristics of complex composition, high temperature, easy condensation of tar, and large dust content, the dust removal technology for volatiles has a certain particularity. This requires the dust collector of coal pyrolysis volatiles has the following characteristics. (1) The dust collector can sustain the high temperature of $>400{ }^{\circ} \mathrm{C}$, and can resist the equipment corrosion by tar steam, $\mathrm{H}_{2} \mathrm{~S}$, and $\mathrm{NH}_{3}$ in the volatiles. (2) In order to avoid the system failure, the temperature of the dust collector should not be lower than the pyrolysis temperature. Otherwise, the condensation of high viscosity tar together with dust will form tar residue and block the piping system. (3) In order to prevent the secondary cracking of tar steam, the 
residence time of volatiles in dust collector should be very short. (4) The collector should keep high dust removal efficiency, so that the dust content in the gas and tar will be decreased. Though the cyclones, scrubbers and electrostatic precipitators are effective method for gas and solid separation, however, these methods are not suitable for the coal pyrolysis volatile which has not only high temperature but also rich tar. ${ }^{15,16}$ In recent years, with the development of the integrated gasification combined cycle (IGCC) and pressurized fluidized bed combustion (PFBC), the granular bed filter (GBF) is increasingly emphasized because of its advantages, ${ }^{17-19}$ such as the high filtering efficiency, stable operating pressure drop, suitable for the high-temperature gas filtration, filter media can be recycled, etc.

Researchers ${ }^{20,21}$ have carried out extensive studies on the separation results of gas and solid in the GBF. Hsu et al..$^{22}$ evaluated the relationship between the filtration performance and a uniform gas flow in a moving granular bed filter (MGBF). The result showed that the filtration efficiency is improved when the gas flow becomes more uniform. In a cross-flow intermittent MGBF, Wenzel et al. ${ }^{23}$ simulated the dust particles in the gaseous flow respectively by glass microspheres and precipitated silica, it was found that the total collection efficiency varies from $82.0 \%$ to $99.8 \%$. Liang et al. ${ }^{11}$ used the nickelbased catalysts as the medium of granular bed filtration and tar catalytic reaction, and preliminarily revealed the interrelationship between dust deposition and the deactivation mechanism of catalyst. However, the research did not concern about the filtration efficiency of the granular bed. When GBF is used for filtration of high temperature and dust-contained volatiles, the dust trapping mechanism of filter media is very complex, involves not only interception, inertial collision, sedimentation, and Brownian diffusion, but also the adsorption and adhesion of dust-contained tar on the surface of the filter media. Therefore, the application of granular bed deep filter in the dust removal of high-temperature coal pyrolysis volatiles has not been reported. Researchers have also proposed many different mathematical models for the GBF theory. ${ }^{24,25}$ However, because the deep filtration of granular bed involves the fluid mechanics theory of two-phase fluid through the porous medium, it is really an extremely complicated physical process. Moreover, the filtration process of GBF has a certain randomness so that the experimental data is difficult to obtain accurately. Therefore, the present reports on the filtration process of GBF can only stay at the semi-empirical and semi-theoretical level. More importantly, the existing researches mainly focus on the mathematical model of cold experiment. The separation behavior of gas and solid in the high-temperature, high-viscosity and dustcontained gas has been rarely reported. The prediction data of industrial dust removal for pyrolysis volatiles is still need to be verified. It is of great significance to investigate the dust removal effect and operation state of GBF in the coal pyrolysis process as well as the model applicability for real pyrolysis volatiles.

This study focused on the dust collection of volatiles in the poly-generation system of CFB combustion/coal pyrolysis. The GBF was applied to the dust removal of coal pyrolysis products by using CFB ash as solid heat carrier. By applying the model calculation of $\mathrm{C}$. Tien et al. ${ }^{26}$ into the lab-scale continuous experiment of the dust removal in GBF, the pressure drop and size distribution of dust particle were examined. Besides, the filtration results at different filter medium, granular bed heights, superficial gas velocities, and medium sizes were discussed, respectively. This work makes a better understanding of the dust removal of pyrolysis volatiles in the poly-generation system of CFB combustion/coal pyrolysis, and may provide the reference for the industrial-scale steady operation of the poly-generation equipment.

\section{Experimental section}

\section{Experimental materials}

Fugu bituminous coal (mean diameter $3 \mathrm{~mm}$ ) from Shaanxi Province is used to carry out the dust removal experiment in the moving-bed pyrolysis system. The proximate and ultimate analyses for Fugu coal are shown in Table 1. Particle size distribution of the raw coal is shown in Table 2. Prior to the experiments, the coal sample was dried at $100{ }^{\circ} \mathrm{C}$ for $6 \mathrm{~h}$. The circulating ash heat carrier was taken from a power plant with a capacity of $35 \mathrm{t} \mathrm{h}^{-1}$ CFB boiler. In the selection of filter medium, the authors found that the semicoke with small size $(\sim 2 \mathrm{~mm})$ is prone to be pulverized during the operation process. Compared with the small quartz sand, the quartz sand with large size has a relatively high price and limited source which makes it almost does not have industrial application value. Therefore, from the perspective of industrial application, the semicoke (mean diameter $8 \mathrm{~mm}$ ) and quarts sand (mean diameter $2 \mathrm{~mm}$ ) were respectively selected as the GBF media in this study, the related parameters are shown in Table 3.

\section{Experimental apparatus and procedures}

The coal pyrolysis experiments by solid heat carrier were performed in the moving bed as shown in Fig. 1. The total height of the apparatus is $\sim 7 \mathrm{~m}$, which is mainly composed of a coal feeder $(\Phi 133 \times 6 \times 1500 \mathrm{~mm})$, a heat carrier feeder $(\Phi 159 \times 6 \times 2000$ $\mathrm{mm})$, a moving-bed pyrolysis reactor $(\Phi 159 \times 6 \times 1800 \mathrm{~mm})$, a material mixer $(\Phi 159 \times 6 \times 600 \mathrm{~mm})$, and a GBF $(\Phi 159 \times 6 \times$ $600 \mathrm{~mm}$ ), etc. Seven temperature measuring points were set up along the bed height of the reactor. The pressure drop of the GBF was observed by the difference of two pressure measuring points which were equipped in the inlet and outlet of the GBF, respectively. Before the test was carried out, a certain amount of ash was added in the moving bed reactor to maintain the material level of the running process. The inert environment of the

Table 1 The proximate and ultimate analyses of Fugu bituminous coal sample

\begin{tabular}{|c|c|c|c|c|c|c|c|c|}
\hline \multicolumn{4}{|c|}{ Proximate analysis/wt\% } & \multicolumn{5}{|c|}{ Ultimate analysis/wt\% } \\
\hline $\mathbf{M}_{\mathrm{ad}}$ & $\mathrm{A}_{\mathrm{ad}}$ & $\mathrm{V}_{\mathrm{ad}}$ & $\mathrm{FC}_{\mathrm{ad}}$ & $\mathrm{C}_{\mathrm{daf}}$ & $\mathrm{H}_{\text {daf }}$ & $\mathrm{N}_{\mathrm{daf}}$ & $\mathrm{S}_{\mathrm{daf}}$ & $\mathrm{O}_{\mathrm{daf}}{ }^{a}$ \\
\hline 10.24 & 6.67 & 33.66 & 49.43 & 76.71 & 6.01 & 0.51 & 15.26 & 1.51 \\
\hline
\end{tabular}

${ }^{a}$ By difference; ad, air dry; daf, dry ash free; M, moisture; A, ash; V, volatile matter; FC, fixed carbon. 
Table 2 Particle size distribution of the raw coal

\begin{tabular}{lllllll}
\hline Size $d_{\mathrm{p}} / \mathrm{mm}$ & $2-3$ & $0.8-2$ & $0.28-0.8$ & $0.125-0.28$ & $0.088-0.125$ & $0.05-0.088$ \\
Weight $w / \%$ & 17.39 & 33.32 & 27.41 & 11.28 & 3.46 & 3.06 \\
\hline
\end{tabular}

Table 3 Characteristics of granular bed filter

\begin{tabular}{|c|c|c|c|c|}
\hline Samples & Density $\left(\mathrm{g} \mathrm{m}^{-3}\right)$ & Sphericity & Average granular size $(\mathrm{mm})$ & Void fraction \\
\hline Quarts sand & $2.65 \times 10^{6}$ & 0.87 & 2 & 0.40 \\
\hline Semicoke & $6.50 \times 10^{5}$ & 0.78 & 8 & 0.45 \\
\hline
\end{tabular}

pyrolysis system was maintained by nitrogen. $10 \mathrm{~kg}$ coal and 40 $\mathrm{kg}$ circulating ash were added to the coal feeder and heat carrier feeder as experimental materials, respectively. After the ash and coal were preheated to $820{ }^{\circ} \mathrm{C}$ and $100{ }^{\circ} \mathrm{C}$ respectively, the two materials were rapidly mixed and fell into the moving-bed pyrolyzer. The moving-bed reactor was heated to make up for the heat loss by electric heating. In order to prevent the condensation of heavy tar in the GBF, the preheating temperature of the GBF was consistent with the reaction temperature. The dust-contained volatile was first purified in the GBF, and then condensed in the water cooler, separated in the gas-liquid separator. Finally, the non-condensable gas entered the gas collector after the measurement of the gas meter, and the tar was fully collected by the tetrahydrofuran rinse of the water cooler, gas-liquid separator and pipelines. The particle size of the trapped dust by GBF and the residual dust in tar was determined in a laser particle size analyzer (Britain, Mastersize 2000). Considering the randomness and repeatability of the dust removal process, each experiment in this work was repeated three times.

\section{Mathematical model}

The calculation method of granular bed filtration proposed by C. Tien et $a .^{27}$ is used in this study. The fixed-bed layer is

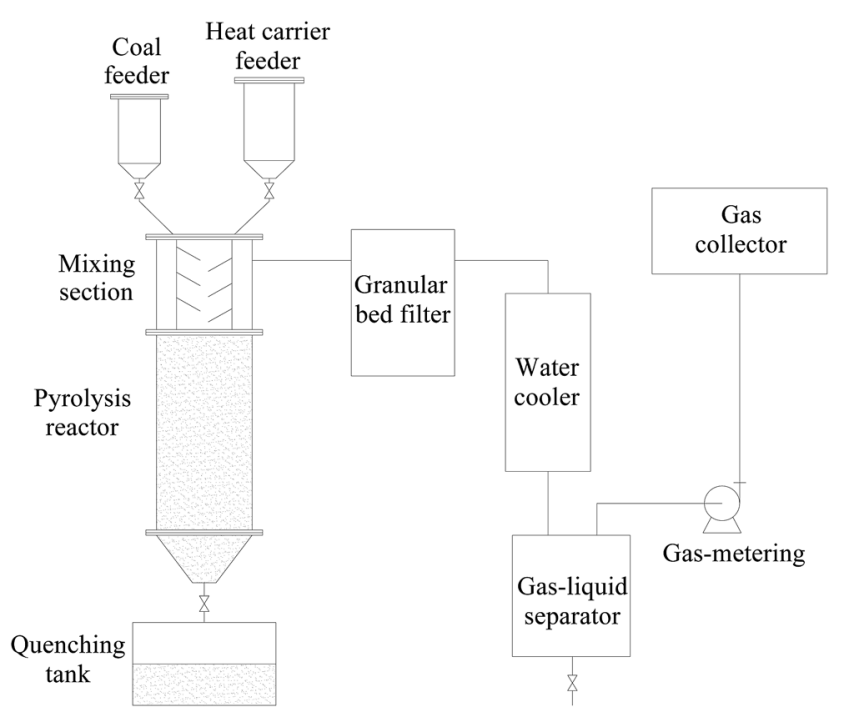

Fig. 1 Schematic diagram of the pyrolysis and dust filtration system. divided into many sequential filter units, and each unit contains a large number of individual filter bodies. Assuming the size of all the individual filter bodies are uniform, then the unit filter efficiency $(\eta)$ is equal to the collection efficiency of the individual filter body. The relation between $\eta$ and the total collection efficiency $(E)$ can be expressed as

$$
E=1-\exp \left(-\frac{H}{d_{\mathrm{g}}}\left(\frac{\pi}{6-6 \varepsilon}\right)^{-\frac{1}{3}} \eta\right)
$$

The unit filter efficiency $\eta$ is constantly changing with the filtering process. The correlations are presented as

$$
\begin{gathered}
F(\alpha, \sigma)=\frac{\eta}{\eta_{0}}=1+\alpha_{1} \sigma^{\alpha_{2}} \\
\alpha_{1}=\left(3.42 \times 10^{-5}+0.292 \times N_{\mathrm{R}}{ }^{1.5}\right) \times N_{\mathrm{St}}{ }^{-3.8} \\
\alpha_{2}=0.26 \ln \left(\frac{1}{N_{\mathrm{St}}}\right)-0.23
\end{gathered}
$$

$$
\left(N_{\mathrm{R}}>0.004,0.001 \leq N_{\mathrm{St}} \leq 0.02\right)
$$

The specific deposit $\sigma$ in eqn (2) is defined as the dust deposition volume in the unit volume of the granular bed. In a fixed-bed filter model, the expression is as follows

$$
\sigma=\frac{1}{V_{\text {bed }}} \int_{0}^{t} \frac{\left(c_{\text {in }}-c_{\text {eff }}\right) u_{\mathrm{g}} S_{\mathrm{g}}}{\rho_{\mathrm{p}}} \mathrm{d} t
$$

Considering the mechanisms of interception and sedimentation as well as the rebound effect of the dust particles, a calculational model of the initial value of unit filter efficiency $\eta_{0}$ can be described by

$$
\begin{gathered}
\eta_{0}=B\left[100 N_{\mathrm{St}}^{2}+0.19\left(4-\frac{4 N_{\mathrm{R}}}{d_{\mathrm{c}}^{*}}+\frac{N_{\mathrm{R}}{ }^{2}}{d_{\mathrm{c}}^{* 2}}\right)^{\frac{1}{2}} \frac{N_{\mathrm{R}}{ }^{1.041}}{d_{\mathrm{c}}^{*}}\right] \\
\left(N_{\mathrm{R}}<0.002, N_{\mathrm{St}}<0.01\right)
\end{gathered}
$$




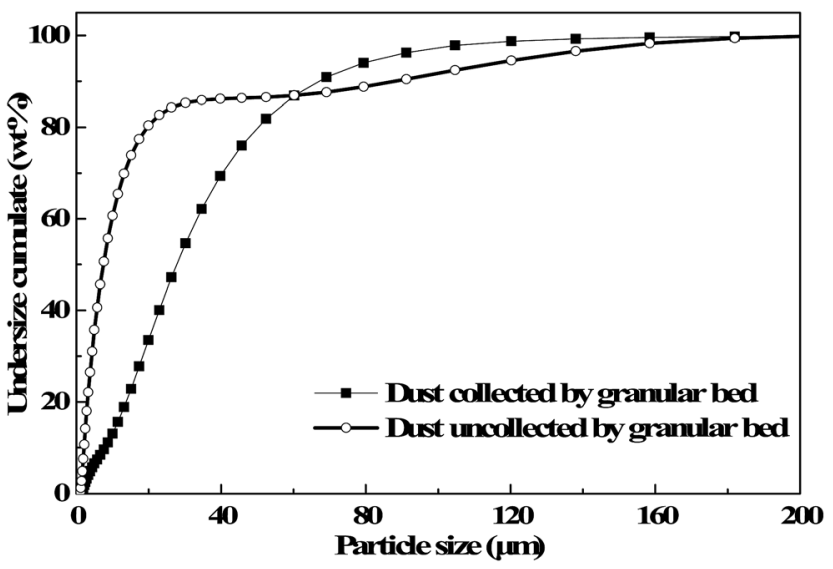

Fig. 2 Size distribution of collected and uncollected dust particles by granular bed (reaction conditions: semicoke particle as filter medium, $T=600{ }^{\circ} \mathrm{C}, H=300 \mathrm{~mm}, \overline{u_{\mathrm{g}}}=0.018 \mathrm{~m} \mathrm{~s}^{-1}$ ).

$$
\begin{gathered}
\eta_{0}=B\left[N_{\mathrm{St}}+0.48\left(4-\frac{4 N_{\mathrm{R}}}{d_{\mathrm{c}}^{*}}+\frac{N_{\mathrm{R}}{ }^{2}}{d_{\mathrm{c}}^{* 2}}\right)^{\frac{1}{2}} \frac{N_{\mathrm{R}}{ }^{1.041}}{d_{\mathrm{c}}^{*}}\right] \\
\left(N_{\mathrm{R}}>0.002, N_{\mathrm{St}}<0.01\right) \\
\eta_{0}=\gamma B\left[N_{\mathrm{St}}+0.48\left(4-\frac{4 N_{R}}{d_{\mathrm{c}}^{*}}+\frac{N_{\mathrm{R}}{ }^{2}}{d_{\mathrm{c}}^{* 2}}\right)^{\frac{1}{2}} \frac{N_{\mathrm{R}}{ }^{1.041}}{d_{\mathrm{c}}^{*}}\right]
\end{gathered}
$$

$$
\left(N_{\mathrm{R}}>0.002, N_{\mathrm{St}} \geq 0.01\right)
$$

where, $B$ is the effect of Reynolds number on the unit filter efficiency, $\gamma$ is an adhesion probability parameter which considered the rebound effect of the dust particles. The correlations ${ }^{28}$ of $B$ and $\gamma$ are respectively presented as

$$
\begin{gathered}
B=7-6 \exp \left(-0.0065 \times N_{\mathrm{Re}}\right) \\
\gamma=0.00318 \times N_{\mathrm{St}}{ }^{-1.248}
\end{gathered}
$$

The eqn (2)-(4) refer to the filtration of monodisperse suspensions. In order to solve eqn (1)-(10), the diameter of dust particles $d_{\mathrm{p}}$ is calculated by the weighted average of particle size distribution in Fig. 2.

\section{Results and discussion}

Dust particle size and operating pressure drop of the GBF

Because the circulating ash and coal particles have a certain size distribution, and part of the coal particles will be broken in the pyrolysis process, the smaller particles are very likely to be taken out of the reactor and then entry into the GBF. One part of the dust particles is collected in the GBF, and the uncollected part is deposited in the cooling system. Fig. 2 shows the size distribution of the collected and uncollected dust particles by the GBF. It can be seen that, the size distribution of the dust particle captured by the GBF is relatively wide. About $95 \mathrm{wt} \%$ of the collected dust particle size is distributed in $0-80 \mu \mathrm{m}$. However, the size distribution of the dust particles uncollected by the GBF is relatively concentrated. The majority particle size of uncollected dust is less than $20 \mu \mathrm{m}$, the mass fraction is $\sim 80 \mathrm{wt} \%$. The result indicates that the particle smaller than $20 \mu \mathrm{m}$ is difficult to be trapped by the GBF. The uncollected dust in the particle size range of $80-160 \mu \mathrm{m}$ accounts for $\sim 10 \mathrm{wt} \%$. The possible reason is that, the uncollected dust is obtained by the tetrahydrofuran dissolution of tar and the separation of filter paper successively. Some insoluble tar macromolecules may be adsorbed on the surface of the dust particle, so that the detected dust particle is slightly larger.

In the running process of the GBF, the pressure drop of quartz sand and semicoke particles as the GBF medium is respectively shown in Fig. 3. It is obvious that the GBF is in a state of non-steady state operation. This is because with the increase of operating time, the amount of deposited dust in the GBF increases gradually, which leads to the increase of the pressure drop in the GBF. Compared with the semicoke particle, the pressure drop of the quartz sand is higher when it is filled as the GBF medium. At the operation time of $22 \mathrm{~min}$, the difference of the pressure drop between the quartz sand and semicoke is $488 \mathrm{~Pa}$. This is mainly related to the smaller particle size and bed void fraction of the quartz sand. In the operations of long-time experiment, an air pump can be set up after the dust removal system to offset part of the pressure drop in the GBF. Therefore, the pressure drop can be stabilized at a lower value and the volatiles can flow out steadily.

\section{Comparison of different filter media on the filter efficiency}

The characteristics of filter media is an important factor that affects the dust removal behavior of the GBF. ${ }^{19}$ Fig. 4 displays the experimental and calculational results of the dust collection by using the quartz sand and semicoke as the filter media. It can be seen that the calculational values of the two filter media

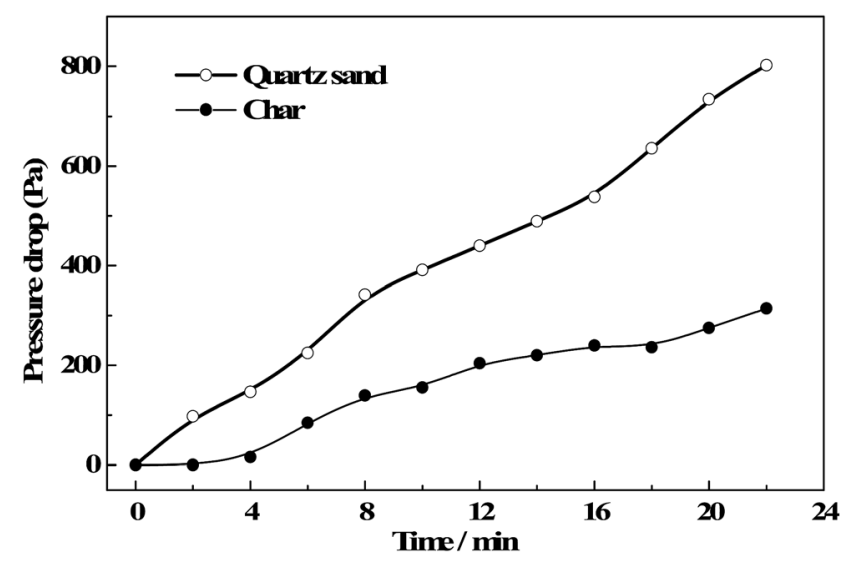

Fig. 3 Effect of particle characteristics on pressure drop of granular bed (reaction conditions: $T=600^{\circ} \mathrm{C}, \mathrm{H}=300 \mathrm{~mm}, \bar{u}_{\mathrm{g}}=0.018 \mathrm{~m} \mathrm{~s}^{-1}$ ). 


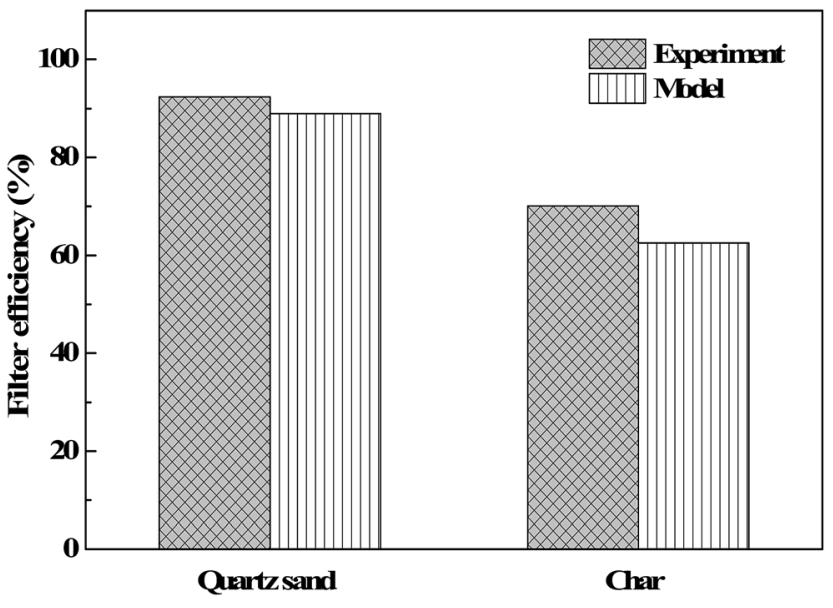

Fig. 4 Effect of particle characteristics on filter efficiency of granular bed (reaction conditions: $T=600^{\circ} \mathrm{C}, \mathrm{H}=300 \mathrm{~mm}, \overline{u_{\mathrm{g}}}=0.018 \mathrm{~m} \mathrm{~s}^{-1}$ ).

efficiency are slightly lower than their corresponding experimental values. After the filtering process, the quarts sand and semicoke after dust cleaning were washed by tetrahydrofuran. It can be seen that the dust coupled with heavy tar flow from the filter media. The tar content from the semicoke was more than that from the quarts sand. Therefore, the difference in the values of the filter efficiency between the model prediction and experimental data can be attributed to two aspects. On the one hand, this is because the filter media particles have a certain specific surface area, so that the sticky tar-adsorbed dust can be adhered to the filter media surface to achieve a better interception effect. On the other hand, the dust particle deposited in the GBF also plays an important role in dust capture. The experimental value and calculational value of the GBF efficiency are respectively $70.03 \%$ and $62.56 \%$ with the semicoke as filter medium. However, the experimental value and calculational value of the GBF efficiency are obviously raised to $92.40 \%$ and $88.96 \%$ when the quartz sand is used as the filter medium. Combined with the results of Fig. 3, although the filter efficiency is better with the quartz sand as the filter medium, but the pressure drop is also higher. In the industrialization process of the CFB combustion/coal pyrolysis poly-generation technology, due to the pyrolysis furnace is operated in a state of micro-positive pressure, it requires lower pressure drop of the GBF to reduce the energy consumption of the system. Therefore, the selection of the filter media is very important for the economic and stability of the GBF operation. Furthermore, in the view of the whole poly-generation process, semicoke discharged from the filter can be directly added into the CFB combustor used as boiler fuel so that the regeneration process will be leaved out. This makes the system easy to operate. Therefore, semicoke is used as the filter medium in the following model calculation and experiment.

By applying the GBF to the dust removal of coal pyrolysis volatiles, fine particle concentration of the gaseous volatile decreases from the filter inlet of $24.37-40.00 \mathrm{~g} \mathrm{Nm}^{-3}$ to the filter outlet of 3.04-9.26 $\mathrm{g} \mathrm{Nm}^{-3}$. When the volatile flows through the condenser, the majority of dust is deposited together with the sticky tar. The dust content in tar product can be reduced to $\sim 1 \%$, while the pyrolysis gas has hardly any dust. Combined with water washing of pyrolysis gas before the desulfurization and denitrification process, the pyrolysis gas can be easily cleaned. For such a CFB combustion/coal pyrolysis polygeneration technology which has great deal of dust in the volatiles, the application of the GBF can reduce dust content both in coal tar and condensation system significantly.

\section{Effect of operating parameters on the filter efficiency}

In this study, the superficial gas velocity of the GBF is adjusted by changing the feed rate of the pyrolysis reactor and then resulting in the volume change of the pyrolysis volatiles. The determination of the superficial gas velocity in the GBF consists of the following assumptions: the non-condensable gas generated by the pyrolysis experiment is approximated as the ideal gas. The contribution of tar vapor to the superficial gas velocity is ignored. Fig. 5a shows the effect of superficial gas velocity on the experiment and model values of the filter efficiency. It can be seen that with the increase of gas velocity, the filter efficiencies of experiment and model prediction both decrease slowly. The effect of the granular bed height on the filter efficiency is shown in Fig. 5b. With the increase of the filter height, the dust removal efficiency of the GBF is significantly improved. When the filter heights are 200,300 and $400 \mathrm{~mm}$, the corresponding experimental values of filter efficiency are $61.08 \%, 70.03 \%$ and $77.88 \%$, and the calculational values of filter efficiency are $49.46 \%, 64.07 \%$ and $74.46 \%$, respectively. Although there are some deviations between the experiment and model values, it can be deduced that the present model can generally reflect the real trend of dust removal efficiency for dust-contained coal pyrolysis volatiles.

\section{Model prediction of filter efficiencies and pressure drop for the optimization of dust removal}

It can be seen in Fig. 5 that the model results of C. Tien et al. can be roughly applied to the coal pyrolysis volatiles dust removal of GBF. Therefore, the model can be used as a reference for the

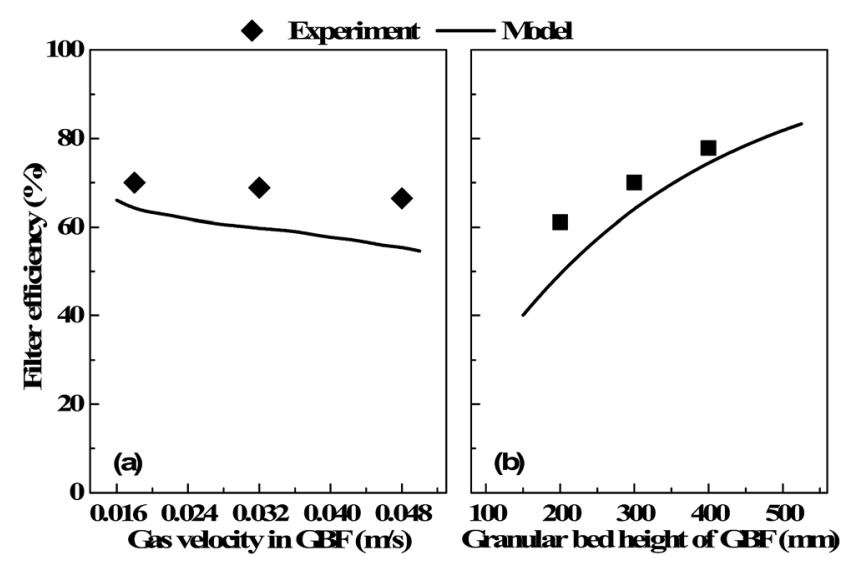

Fig. 5 Effect of (a) superficial gas velocity and (b) granular bed height on filter efficiency of GBF (reaction conditions: (a) $T=600^{\circ} \mathrm{C}, \mathrm{H}=300$ $\mathrm{mm}$; (b) $T=600^{\circ} \mathrm{C}, \bar{u}_{\mathrm{g}}=0.018 \mathrm{~m} \mathrm{~s}^{-1}$ ). 
optimization and amplification of dust removal in GBF. Fig. 6a shows the effect of particle size on the filter efficiency and pressure drop. When the semicoke size decreases from $10 \mathrm{~mm}$ to 6 $\mathrm{mm}$, the filtration efficiency of GBF significantly increases from $58.77 \%$ to $90.90 \%$. Furthermore, when the semicoke size decreases from $6 \mathrm{~mm}$ to $1 \mathrm{~mm}$, the filtration efficiency smoothly increases from $90.90 \%$ to $100 \%$. This is due to the fact that the smaller semicoke size has decreased Reynolds number $\left(N_{\mathrm{Re}}\right)$ and increased intercept parameter $\left(N_{R}\right)$ at the same time. The viscosity effect of dust-contained fluid is more significant, and the intercepting effect of filter media is strengthened. Otherwise, due to the increase of Stocks number $\left(N_{\mathrm{St}}\right)$, the streamline motion paths of dust particles evolve into curves gradually. Dust is much easier to bypass the medium obstacles. Therefore, considering the above two aspects, the influencing degree of particle size on the filtration efficiency and pressure drop are weakened when the particle size is less than $6 \mathrm{~mm}$. However, as the particle size decreases from $10 \mathrm{~mm}$ to $1 \mathrm{~mm}$, the bed pressure drop increases from 114.3 $\mathrm{Pa}$ to $1391.7 \mathrm{~Pa}$. As a result, it is more appropriate to select the semicoke size of about $6 \mathrm{~mm}$ when take into account both filtration efficiency and bed pressure.

As shown in Fig. 6b, when the granular bed height is below $400 \mathrm{~mm}$, the filter efficiency and operating pressure drop both increase with the bed height significantly. However, when the granular bed height is higher than $400 \mathrm{~mm}$, the increasing trend of filter efficiency becomes smooth and the operating pressure drop still increases in a straight line. Thus, within the reasonable range of the operation pressure drop in the polygeneration technology of CFB combustion/coal pyrolysis, increasing the filter height is beneficial to reduce the dust content of the pyrolysis products. According to the model prediction result, the granular bed height of $400 \mathrm{~mm}$ is appropriate. The corresponding filter efficiency and operating pressure drop are $90.90 \%$ and $193.57 \mathrm{~Pa}$, respectively.

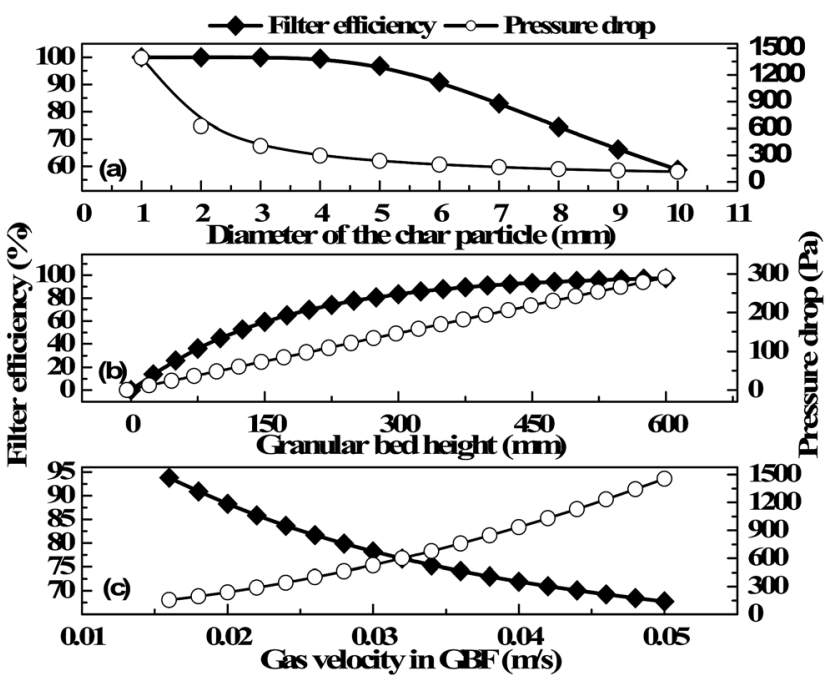

Fig. 6 Model prediction of filter efficiencies and pressure drop at different (a) semicoke sizes; (b) granular bed height; (c) superficial gas velocity (model parameters: (a) $T=600^{\circ} \mathrm{C}, \overline{u_{\mathrm{g}}}=0.018 \mathrm{~m} \mathrm{~s}^{-1}, \mathrm{H}=400$ $\mathrm{mm}$; (b) $T=600^{\circ} \mathrm{C}, \bar{u}_{\mathrm{g}}=0.018 \mathrm{~m} \mathrm{~s}^{-1}, d_{\mathrm{g}}=6 \mathrm{~mm}$; (c) $T=600^{\circ} \mathrm{C}, d_{\mathrm{g}}=$ $6 \mathrm{~mm}, \mathrm{H}=400 \mathrm{~mm}$ ).
Fig. $6 c$ shows the effect of superficial gas velocity on the filter efficiencies and pressure drop. It can be seen that the slower superficial gas velocity is conducive to the dust collection. This is mainly due to the fact that the increase of superficial gas velocity can promote the inertial collision effect between the filter medium particles and the dust particles, but meanwhile the Reynolds number $\left(N_{\mathrm{Re}}\right)$ will be improved at a faster superficial gas velocity then cause a sharper collision rebound effect which is not beneficial to the dust collection. Otherwise, the faster superficial gas velocity will blow out more dust from the reactor and destruct the contact state of the GBF medium. Part of the collected dust is raised again, then resulting in a heavier dust load. As a result, the filter efficiency of the GBF decreases with the increase of superficial gas velocity.

In the model calculation, it was found that the applicable range of the Stocks number $N_{\mathrm{St}}$ in eqn (2)-(4) is particularly narrow. The $N_{\text {St }}$ at the superficial gas velocity of $0.018,0.032$, and $0.048 \mathrm{~m} \mathrm{~s}^{-1}$ are in the range of $0.001 \leq N_{\mathrm{St}} \leq 0.02$. However, when the superficial gas velocity increases slightly, the $N_{\mathrm{St}}$ will exceed the range and then result in a larger difference of filtration efficiency between the experimental and model values. Therefore, based on the experimental values, the model could be improved by the parameter calibration of $\alpha_{1}$ and $\alpha_{2}$ in eqn (2). Firstly, plotting the value of $\ln (F(\alpha, \sigma)-1)$ versus $\ln \sigma . \alpha_{1}$ and $\alpha_{2}$ can be obtained from the intercept and slope of above plotting, respectively. Secondly, combined with the values of $\alpha_{1}$ and $\alpha_{2}$ at different $N_{\text {St }}$, the coefficient of eqn (3) and (4) can be obtained. Therefore, the applicability of eqn (2)-(4) can be optimized according to the specific experiments.

\section{Conclusions}

The dust removal of pyrolysis volatiles is one of the technical bottlenecks in the industrialization process of the CFB combustion/coal pyrolysis. This study applied the GBF to CFB combustion combined with coal pyrolysis system. By carrying out the lab-scale moving-bed coal pyrolysis experiment, the model applicability for the real pyrolysis volatiles which has high temperature, high viscosity, and a large amount of dust was verified. The dust removal characteristics of the GBF at different filter media, granular bed heights, superficial gas velocities, and medium sizes were investigated, respectively. The major conclusions are summarized as follows:

The dust in the particle size range of $0-20 \mu \mathrm{m}$ is difficult to be trapped by the GBF. With the increase of operating time, the pressure drop of the GBF increases gradually. The higher filter height, slower superficial gas velocity, and smaller medium size are conducive to decrease the dust content in the pyrolysis volatiles. Because of the sticky tar-adsorbed dust is inclined to adhere to the filter media surface, the prediction value of the filter efficiency is slightly lower than the experimental value. The C. Tien model results of the initial filtration efficiency can be used as the selection reference of the medium species and design parameters for GBF. This study can provide reference for the industrial-scale design and development of the dust removal technology in the poly-generation system of CFB combustion/coal pyrolysis in the future. 


\section{Nomenclature}

\section{Abbreviations}

$\begin{array}{ll}\text { ad } & \text { Air-dried basis } \\ \text { CFB } & \text { Circulating fluidized bed } \\ \text { daf } & \text { Dry and ash free basis } \\ \text { GBF } & \text { Granular bed filter } \\ \text { MGBF } & \text { Moving granular bed filter } \\ \text { PFBC } & \text { Pressurized fluidized bed combustion } \\ \text { IGCC } & \text { Integrated gasification combined cycle } \\ \text { wt } \% & \text { Weight percentage }\end{array}$

\section{Latin letters}

B

$C_{\mathrm{s}} \quad$ Drag coefficient, defined by $C_{\mathrm{s}}=1+2 \lambda / d_{\mathrm{p}}(1.257$

$\left.+0.4 \exp \left(-0.55 d_{\mathrm{p}} / \lambda\right)\right)$

Dust concentration of the GBF inlet, $\mathrm{g} \mathrm{Nm}^{-3}$

Dust concentration of the GBF outlet, $\mathrm{g} \mathrm{Nm}^{-3}$

Dimensionless constriction diameter

Diameter of filter medium, $\mathrm{m}$

Diameter of dust particle, $\mathrm{m}$

Total collection efficiency

Function defined by eqn (2)

Bed height, $\mathrm{m}$

Intercept parameters, defined by $N_{\mathrm{R}}=d_{\mathrm{p}} / d_{\mathrm{g}}$

Reynolds number, defined by $N_{\mathrm{Re}}=d_{\mathrm{g}} u_{\mathrm{g}} \rho_{\mathrm{g}} / \mu$

Stocks number, defined by $N_{\text {Stock }}=\rho_{\mathrm{p}} d_{\mathrm{p}}{ }^{2} u_{\mathrm{g}} C_{\mathrm{s}} /$

$9 \mu d_{\mathrm{g}}$

Cross-sectional area of the gas passing through

the bed, $\mathrm{m}^{2}$

Filtration time, $\mathrm{s}$

Pyrolysis temperature, $\mathrm{K}$

Average superficial gas velocity, $\mathrm{m} \mathrm{s}^{-1}$

Fixed bed volume, $\mathrm{m}^{3}$

\section{Greek letters}

$\begin{array}{ll}\alpha_{1} & \text { Vector parameter in function } F(\alpha, \sigma) \\ \alpha_{2} & \text { Vector parameter in function } F(\alpha, \sigma) \\ \gamma & \text { Adhesion probability parameter } \\ \varepsilon & \text { Void fraction of bed } \\ \eta & \text { Unit filter efficiency, \% } \\ \eta_{0} & \text { Initial value of } \eta, \% \\ \lambda & \text { Mean free path of gas molecules, defined by } \lambda= \\ \mu & 2.9685 \times 10^{-5} T /(P(1+110 / T)), \mathrm{m} \\ \rho_{\mathrm{g}} & \text { Fluid viscosity, } \mathrm{g}(\mathrm{m} \mathrm{s})^{-1} \\ \rho_{\mathrm{p}} & \text { Gas density, } \mathrm{g} \mathrm{m}^{-3} \\ \sigma & \text { Particle density, } \mathrm{g} \mathrm{m}^{-3}\end{array}$

\section{Acknowledgements}

The work was supported by the National Natural Science Foundation of China (Grant No. 21376142).

\section{References}

1 H. F. Guo, Q. X. Cheng, Z. Jin, D. Wang, G. W. Xu and Y. Y. Liu, $R S C A d v .$, 2016, 6, 102231-102248.

2 Y. J. He, F. Z. Xiao and Z. H. Luo, Powder Technol., 2015, 274, 28-36.

3 Y. W. Wang, W. X. Xi, X. L. Huai, X. F. Li and J. Cai, Ann. Nucl. Energy, 2015, 85, 418-425.

4 G. Voitic and V. Hacker, RSC Adv., 2016, 6, 98267-98296.

5 M. Eriksson and M. R. Golriz, Int. J. Therm. Sci., 2005, 44, 399-409.

6 P. Liang, W. M. Jiang, Y. Q. Zhang, X. H. Wang and J. L. Zhu, Fuel Process. Technol., 2016, 150, 16-22.

7 J. M. Cen, M. X. Fang, Q. H. Wang, Z. Y. Luo and K. F. Cen, Chem. Ind. Eng. Prog., 2011, 30, 88-93.

8 P. Liang, Y. Q. Zhang, W. M. Jiang, A. F. Wei, T. Liu and J. F. Wu, Energy Fuels, 2015, 29, 7727-7733.

9 X. Qu, P. Liang, Z. F. Wang, R. Zhang, D. K. Sun, X. K. Gong, Z. X. Gan and J. C. Bi, Chem. Eng. Technol., 2011, 34, 61-68.

10 Y. Q. Zhang, J. L. Zhu, X. H. Wang, X. W. Zhang, S. X. Zhou and P. Liang, Fuel Process. Technol., 2016, 154, 227-234.

11 P. Liang, Y. Q. Zhang, A. F. Wei, X. H. Wang and T. Liu, Energy Fuels, 2015, 29, 70-77.

12 I. A. El-Hedok, L. Whitmer and R. C. Brown, Powder Technol., 2011, 214, 69-76.

13 G. Xiao, X. H. Wang, J. P. Zhang, M. J. Ni, X. Gao, Z. Y. Luo and K. F. Cen, Powder Technol., 2013, 244, 93-99.

14 S. Heidenreich, Fuel, 2013, 104, 83-94.

15 Y. S. Chen, Y. P. Chyou and S. C. Li, Appl. Therm. Eng., 2014, 74, 146-155.

16 S. I. Yang, I. L. Chung and S. R. Wu, Powder Technol., 2012, 228, 121-127.

17 T. D. Hadley, K. S. Lim, J. Orellana, R. R. Denke, K. Chiang and N. R. Burkeb, Chem. Eng. Res. Des., 2012, 90, 52-62.

18 J. T. Zhao, J. J. Huang, J. H. Wu, Y. T. Fang and Y. Wang, Powder Technol., 2008, 180, 2-8.

19 C. A. P. Zevenhoven, K. R. G. Hein and B. Scarlett, Filtr. Sep., 1993, 30, 550-553.

20 C. J. Hsu, S. S. Hsiau, Y. S. Chen and J. Smid, Adv. Powder Technol., 2010, 21, 614-622.

21 Y. S. Chen, S. S. Hsiau, S. C. Lai, Y. P. Chyou, H. Y. Li and C. J. Hsu, J. Hazard. Mater., 2009, 171, 987-994.

22 C. J. Hsu and S. S. Hsiau, Powder Technol., 2015, 274, 20-27. 23 B. M. Wenzel, C. B. Porciúncula, N. R. Marcilio, H. B. Menegolla, G. M. Dornemann, M. Godinho and C. B. Martins, Sep. Purif. Technol., 2014, 133, 108-119.

24 K. Ushiki and C. Tien, Powder Technol., 1989, 58, 243-258.

25 Y. Jung, S. A. Walata and C. Tien, Aerosol Sci. Technol., 1989, 11, 168-182.

26 C. Tien, Granular Filtration of Aerosols and Hydrosols, Butterworth Publishers, Boston, USA, 1989, pp. 177-215.

27 T. Takahashi, S. A. Walata and C. Tien, AIChE J., 1986, 32, 684-690.

28 H. Yoshida and C. Tien, AIChE J., 1985, 31, 1752-1754. 